\title{
Vérité et justice comme remèdes au trauma : Bloody Sunday et l'enquête Saville
}

\section{Charlotte Barcat}

\section{Q OpenEdition \\ 12 Journals}

\section{Édition électronique}

URL : http://journals.openedition.org/etudesirlandaises/2154

DOI : 10.4000/etudesirlandaises. 2154

ISSN : 2259-8863

\section{Éditeur}

Presses universitaires de Rennes

\section{Édition imprimée}

Date de publication : 30 juin 2011

Pagination : 91-106

ISBN : 978-2-7535-1348-8

ISSN : 0183-973X

\section{Référence électronique}

Charlotte Barcat, "Vérité et justice comme remèdes au trauma : Bloody Sunday et l'enquête Saville ", Études irlandaises [En ligne], 36-1 | 2011, mis en ligne le 30 juin 2013, consulté le 02 mai 2019. URL: http://journals.openedition.org/etudesirlandaises/2154; DOI : 10.4000/etudesirlandaises.2154

Ce document a été généré automatiquement le 2 mai 2019.

(c) Presses universitaires de Rennes 


\title{
Vérité et justice comme remèdes au trauma: Bloody Sunday et l'enquête Saville
}

\author{
Charlotte Barcat
}

1 Bloody Sunday s'apparente à un traumatisme à plusieurs niveaux: si l'on comprend le trauma comme une blessure aux effets durables, on peut considérer qu'il fut d'abord infligé, bien sûr, aux personnes directement touchées par le drame: aux blessés, aux familles des victimes, et aux témoins ; mais également de façon plus abstraite, au niveau de la mémoire collective, et en particulier de la perception du gouvernement par la communauté nationaliste.

2 Comme le souligne Eamonn McCann, les circonstances très particulières dans lesquelles s'est déroulé Bloody Sunday lui ont conféré un potentiel traumatique très important :

La plupart des homicides dans le Nord, comme toujours dans les conflits de ce genre, se produisaient avec la rapidité de l'éclair, dans des rues isolées ou en pleine nuit, généralement lors d'une embuscade furtive ou de l'explosion d'une bombe dissimulée. Bloody Sunday s'est déroulé sur une période de peut-être dix minutes, dans une zone urbanisée en plein jour et dans des circonstances où des milliers d'amis et de voisins de victimes étaient massés dans les environs immédiats ${ }^{1}$.

3 En effet, la communauté des habitants du Bogside étant très soudée et comprenant beaucoup de familles nombreuses, la mort de treize personnes affecta directement un très grand nombre de gens. À ce groupe, il faut également ajouter les blessés et les personnes qui faisaient partie des manifestants, ou habitaient dans les immeubles alentour, tous susceptibles d'avoir subi un traumatisme. La persistance du trauma était d'autre part favorisée par le fait que les événements s'étaient déroulés dans un endroit qui faisait partie de la vie quotidienne des habitants du Bogside : beaucoup d'entre eux habitaient les Rossville Flats, ces grands immeubles aujourd'hui détruits, aux pieds desquels avait eu lieu la fusillade. Dans son article sur le lien entre trauma et lieux géographiques, l'historien Graham Dawson insiste sur cet aspect : 
Les sites de cet événement atroce sont - ou étaient - des lieux géographiques réels $\mathrm{du}$ Bogside, où la vie de tous les jours s'était déroulée avant, et continuait à se dérouler après Bloody Sunday. Après ces événements, cependant, ces endroits devinrent également - et sont restés - des sites de mémoire et de traumatisme dans un paysage culturel transformé 2 .

Bloody Sunday peut donc être vu comme un événement qui a traumatisé une communauté toute entière, mais il a aussi infligé un traumatisme au niveau de la mémoire collective, et en particulier de la confiance en l'État du côté de la communauté nationaliste, qui était déjà mal intégrée dans l'État nord-irlandais. Le juriste Dermot Walsh affirme :

Bloody Sunday peut être cité, avec l'internement et les grèves de la faim dans les $\mathrm{H}$ blocks, comme un des événements qui a le plus contribué à provoquer l'aliénation des nationalistes vis-à-vis de l'État d'Irlande du Nord ${ }^{3}$.

Plusieurs témoignages montrent comment les réactions d'horreur devant Bloody Sunday ont influencé la perception identitaire des nationalistes, en renforçant encore l'image d'une minorité en lutte contre un État britannique illégitime. Graham Dawson résume le phénomène en parlant de " cassure identitaire ", et explique :

Les catholiques de Derry s'éloignèrent d'une identification souvent ambivalente avec la Grande-Bretagne pour aller vers une identification qui se construisait en opposition ouverte avec elle, en tant que cibles et victimes irlandaises de la puissance militaire britannique; ce qui eut pour effet de renforcer le sentiment d'appartenance à une identité nationale irlandaise et de causer l'aliénation vis-à-vis des fondations de l'État britannique en Irlande du Nord 4 .

6 Il cite comme exemple les propos de Nigel Cooke, qui avait participé à la manifestation pour les droits civiques le jour de Bloody Sunday alors qu'il était adolescent :

Bloody Sunday eut un impact très profond sur moi [...]. S'il y eut un moment décisif où j'ai cessé de me penser comme étant plus ou moins britannique, ce fut celui-là. Parce que j'ai immédiatement compris qu'une telle atrocité n'aurait jamais pu être envisagée ailleurs en Grande-Bretagne. Des citoyens anglais, gallois ou écossais n'auraient jamais pu être classés sans distinction parmi les "ennemis" de l'armée britannique. Seul les Irlandais pouvaient tenir ce rôle ${ }^{5}$.

7 On retrouve les mêmes thèmes de la perte de toute forme d'identification à l'État britannique et de renforcement d'une identité irlandaise construite en opposition à cet État chez Don Mullan, témoin de Bloody Sunday devenu activiste dans le domaine de l'humanitaire et auteur du livre Eyewitness Bloody Sunday: The Truth :

Bloody Sunday fut le début de la fin du colonialisme britannique dans mon cœur et dans mon âme. [...] Le moindre espoir d'acheter ma loyauté à la couronne britannique par des réformes superficielles mourut ce jour-là. Mon identité devint très clairement définie. Je suis Irlandais, pas Nord-irlandais. La partition a échoué pour toujours ${ }^{6}$

8 L'implication des forces de l'État, institution qui est censée protéger ses citoyens, était sans aucun doute l'un des facteurs qui donna à cet événement un tel potentiel traumatique. L'incapacité de l'appareil d'État à répondre à la demande de justice dans les mois qui suivirent fut tout aussi importante : le rapport Widgery fut vécu comme un second traumatisme.

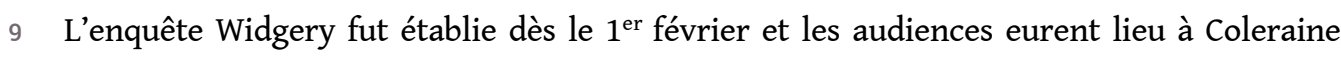
entre le 14 février et le 20 mars. Le choix de Coleraine était déjà, en soi, une maladresse : cette petite ville située au Nord de Derry était connue pour sa majorité unioniste, ce qui ne pouvait manquer de donner aux témoins issus de la communauté nationaliste l'impression d'un manque d'objectivité. Le rapport qui fut publié le 18 avril ne fit que 
confirmer leurs craintes: bien qu'admettant qu'il n'y avait aucune preuve que les personnes tuées par les soldats le 30 janvier avaient manipulé des armes à feu ou des bombes, Widgery laissait planer le doute en précisant que de "forts soupçons ${ }^{7}$ » subsistaient concernant certains d'entre eux. Plus important encore, la conclusion de son rapport faisait porter à NICRA la plus grande part de responsabilité dans la tragédie, affirmant :

Il n'y aurait pas eu de morts à Londonderry le 30 janvier si ceux qui avaient organisé la manifestation illégale n'avaient pas ce faisant créé une situation extrêmement dangereuse dans laquelle un affrontement entre les manifestants et les forces de sécurité était presque inévitable ${ }^{8}$.

10 Le rapport réussit en partie à apaiser les esprits, du moins au sein de l'opinion publique britannique ${ }^{9}$, mais l'enquête Widgery fut vite baptisée le «Widgery Whitewash » par ceux qui contestaient cette version officielle, le processus tout entier étant perçu comme une tentative de «blanchir » l'armée de la part du gouvernement. Les nombreux livres de témoignages publiés dans les années 1990 montrent que les familles des victimes considèrent souvent le rapport Widgery comme une " seconde mort » pour leurs proches, ou comme « l'insulte ultime » de la part du gouvernement ${ }^{10}$. Joelle Gartner cite les propos d'un(e) des proches des victimes, qui reste anonyme, concernant les effets du rapport Widgery sur ceux qui avaient encore foi en l'État :

Une onde de choc a traversé la ville le jour où le rapport Widgery a été publié, une véritable onde de choc. Le refus d'admettre la vérité a fait en réalité plus de mal au niveau psychologique [...] que les morts elles-mêmes, parce que naïvement, dans un sens, nous attendions mieux ${ }^{11}$.

11 Les membres des familles des victimes évoquent souvent le rapport en des termes qui évoquent le meurtre, l'assassinat, de la vérité ou de la justice. John Kelly, à la tête de la Bloody Sunday Justice Campaign (BSJC), dit avoir toujours répété que « la quinzième victime de Bloody Sunday a été la vérité ${ }^{12}$ ». Don Mullan, quant à lui, parle "d'assassinat de la justice », et affirme: "Les Paras avaient assassiné des civils irlandais qui n'étaient pas armés ; Lord Widgery a assassiné la vérité ${ }^{13}$.»

Dans les années qui suivirent, la mémoire de Bloody Sunday fut entretenue notamment grâce à la marche de commémoration, qui a lieu chaque année depuis 1972, puis grâce à un certain nombre d'organisations fondées par des familles de victimes ou des activistes des droits de l'Homme. La Bloody Sunday Initiative fut fondée en 1989 : cette association de tradition nationaliste militait en faveur de la réconciliation, ainsi que d'une nouvelle enquête sur Bloody Sunday. En avril 1992, des proches des victimes s'associèrent pour créer la BSJC, avec trois objectifs : une reconnaissance publique et sans équivoque par le gouvernement britannique de l'innocence des victimes de Bloody Sunday, une répudiation publique du rapport Widgery, et des poursuites judiciaires.

13 Malgré la redécouverte dans les années 1990 de nombreux documents ignorés par la première enquête, et la publication de plusieurs ouvrages contestant la version des faits de Widgery, le gouvernement conservateur de John Major refusa systématiquement l'idée d'une nouvelle enquête. Le sujet fut évoqué à plusieurs reprises durant les débats parlementaires ${ }^{14}$, avec à chaque fois une réponse similaire: le gouvernement estimait qu'il n'y avait «à sa connaissance aucune raison » de rouvrir l'enquête, réaffirmait sa confiance dans le rapport Widgery, et ajoutait qu'il était inutile de « ressasser de vieux problèmes ${ }^{15} »$. 
14 Le changement de gouvernement avec l'arrivée des travaillistes sous Tony Blair en mai 1997 permit d'accélérer considérablement le processus. Sous la pression du gouvernement irlandais de Bertie Ahern, qui avait fait connaître son intention de publier un rapport critiquant l'enquête Widgery à la lumière des nouveaux éléments récemment découverts, Tony Blair fit le 29 janvier 1998 une déclaration à la Chambre des Communes qui annonçait l'établissement d'une nouvelle enquête sur Bloody Sunday. Celle-ci, tout comme l'enquête Widgery, serait régie par le Tribunal of Inquiry (Evidence) Act de 1921, et serait confiée à Lord Saville, associé à deux juges venus de pays du Commonwealth.

Dans le contexte d'une enquête sur un événement tel que Bloody Sunday, montrer que l'on reconnaît l'expérience traumatisante vécue par les victimes et les témoins est fondamental. Pour David A. Crocker, auteur d'un article intitulé «Truth Commissions, Transitional Justice, and Civil Society ", cet aspect est indispensable à toute transition :

[...] on doit offrir aux victimes ou à leurs familles une tribune pour qu'ils racontent leur histoire et que leur témoignage soit reconnu publiquement. Quand les victimes sont en mesure de donner leur version des événements et reçoivent de la sympathie pour leurs souffrances, elles sont respectées en tant que personnes et sont traitées avec dignité plutôt que - comme c'était le cas auparavant - avec mépris ${ }^{16}$.

16 L'utilité d'une enquête publique telle que l'enquête Widgery est de fournir une telle tribune. Or, la possibilité de « raconter leur histoire » est précisément ce qui a été refusé à beaucoup de personnes affectées par Bloody Sunday lors de cette première enquête. Un des principaux reproches faits à Widgery concerne en effet sa décision de ne pas tenir compte de certains témoignages, ainsi que sa propension à donner plus de crédit à la parole des soldats qu'à celle des témoins civils. Kay Duddy fait allusion à ce sentiment de rejet : « Le tribunal Widgery a tellement limité le nombre de témoins que les gens n'ont pas eu la chance, l'occasion de s'exprimer devant le tribunal pour raconter ce qu'ils avaient $\mathrm{vu}^{17}$. » On peut remarquer qu'il utilise parfois en citant certain témoins civils des guillemets, ou des formules comme « il était convaincu que... » qui suggèrent une certaine distanciation, voire l'incrédulité, ce qu'il ne fait pas lorsqu'il rapporte les témoignages des soldats. On trouve un exemple de cela dans son résumé des propos du prêtre Edward Daly : "Le père Daly a continué de courir et après quelques mètres il a entendu "une fusillade", "un très grand nombre de coups de feu" [...]. Il était convaincu que tous les tirs venaient de derrière et pensait que le reste de la foule croyait aussi que c'était le cas ${ }^{18}$. " Au contraire, il accepte souvent de façon assez arbitraire les justifications des soldats : " Il n'y a aucune raison de supposer que les soldats auraient ouvert le feu s'ils n'avaient pas d'abord été la cible de $\operatorname{tirs}^{19}$. $\gg$ Peter Pringle, journaliste, dénonce un des arguments utilisés pendant l'enquête Widgery dans son traitement de certains témoignages de civils :

Widgery a dit que le témoignage des civils, qui n'avaient pas vu de bombes à clous, ne constituait que des témoignages «négatifs », et qu'on ne peut pas prouver par la négative. Mais, comme l'a écrit Bryan McMahon dans sa critique de Widgery en 1974, « comment les civils pouvaient-ils prouver qu'il n'y avait pas de bombes autrement qu'en déclarant qu'ils n'en avaient pas vu ${ }^{20}$ ?»

17 Le fait que tant de témoignages, de blessés mais aussi de nombreux civils souhaitant collaborer aient été ignorés ou discrédités donnèrent à la communauté traumatisée l'impression que ses récits, ses "vérités", n'avaient pas été pris en compte par le tribunal.

Cela eut pour conséquence l'apparition d'une mémoire parallèle, non-officielle, de Bloody Sunday. Les livres de témoignages écrits à partir des années 1990 en sont un exemple : Trisha Ziff, éditrice du livre qui porte le nom de Hidden Truths, explique qu'en publiant ce 
recueil de témoignages et de photographies, elle participe au «processus de guérison ${ }^{21}$ ", reprenant l'image d'un trauma ou d'une blessure à soigner. Ce livre fut réalisé à partir d'une exposition photographique éponyme, dont Trisha Ziff disait en 2002 : «L'œil de la caméra a tout saisi. [...] Les gens devaient voir Bloody Sunday. Tout voir. Et c'est ce que nous avons ici. Des vérités qui ne sont plus cachées ${ }^{22}$. »Cet insistance sur la " vérité » du document photographique et du témoignage, deux types de représentation du passé qui apparaissent comme "brutes", sans médiation, est assez typique de la mémoire nonofficielle de Bloody Sunday. On la retrouve dans le livre de Don Mullan, Eyewitness Bloody Sunday: The Truth, ainsi que dans les livres d'Eamonn McCann, Bloody Sunday in Derry: What Really Happened et The Bloody Sunday Inquiry: The Families Speak Out. Le livre de Don Mullan, publié en 1997, reprenait des centaines de témoignages recueillis après Bloody Sunday par NICRA, que Widgery avait refusé de considérer en arguant du fait qu'ils avaient été soumis trop tard et n'apportaient aucun élément nouveau ${ }^{23}$. Mullan redécouvrit fin 1995 ces témoignages, qui contenaient en réalité des éléments absents du rapport, susceptibles de remettre en cause l'analyse de Widgery, et décida de les publier.

Tous ces ouvrages ont en commun l'utilisation extensive de témoignages cités directement, l'auteur se contentant souvent d'écrire l'introduction ou quelques chapitres intercalaires, sans ajouter de commentaires dans les parties consacrées aux propos des personnes interrogées. Ils ont également en commun une revendication de vérité : on voit de par leurs titres qu'ils envisagent leurs ouvrages comme des "livres-révélations », qui dévoilent la vérité que la version officielle aurait cachée ou déformée. Là où les représentants de l'État inspirent méfiance, les «gens de la rue », les habitants de Derry, sont vus comme les gardiens d'une vérité authentique. Quelles que soient les réserves que l'on puisse avoir vis-à-vis de cette idéalisation du témoignage, il est certain que la réintégration dans la version officielle de tous ces "fragments de vérité ", auparavant rejetés et relégués à une position de mémoire dissidente, est un des objectifs principaux de l'enquête Saville, afin d'atténuer le sentiment d'injustice ressenti par la communauté du Bogside et par tous ceux qui s'identifient à leur expérience.

Le caractère officiel du processus est donc fondamental: le besoin de "raconter ses histoires » est aussi un besoin de reconnaissance. Une des questions qui a suscité des interrogations concernait la possibilité pour le gouvernement britannique de régler le problème en présentant des excuses, comme l'a fait Tony Blair concernant la mauvaise gestion de la «Grande Famine » de 1845-1849 par l'État britannique ${ }^{24}$. Jonathan Powell, qui dévoile dans son livre Great Hatred, Little Room: Making Peace in Northern Ireland les coulisses du processus de paix auquel il a participé en tant que chef de cabinet de Tony Blair, raconte :

Le problème a été soulevé début 1998, lorsque Mo [Mowlam, secrétaire d'état à l'Irlande du Nord] a écrit à Tony au sujet de la nécessité d'une enquête. Tony et moi pensions tous les deux que des excuses seraient suffisantes. Mais les Irlandais nous avaient dit que des excuses ne suffiraient pas et qu'il faudrait mettre en place une enquête indépendante ${ }^{25}$.

Du côté des familles, cette idée de présenter des excuses était plutôt ressentie comme une expression de mépris. Eileen Greene, veuve de Paddy Doherty, affirmait à Joanne O'Brien :

Nous ne cherchons pas à obtenir des excuses. On ne s'excuse que lorsqu'on a fait quelque chose de mal, et selon le gouvernement britannique, ça n'était pas le cas. [...] Nous méritons plus que ça. Aux yeux de certains, nos proches sont toujours des tireurs ou des lanceurs de bombes ${ }^{26}$. 
Joelle Gartner, qui a interrogé plusieurs membres des familles à ce sujet, affirme : «Les gens ont besoin de vérité, pas d'excuses, [qui] seraient vues comme une insulte et une tentative de désamorcer la demande de vérité ${ }^{77}$ ». Les excuses peuvent en effet être perçues comme une solution facile, une sorte de "politique du geste » qui permet de se débarrasser d'un problème à peu de frais ${ }^{28}$. Par contraste, le fait de rouvrir une enquête similaire à la première est très significatif : bien que le gouvernement britannique ait refusé de la représenter comme telle, l'enquête Saville est bien une remise en cause de l'enquête Widgery, ce qui signifie que cette dernière a été désavouée par le mécanisme même qui l'avait engendrée.

Le rapport Saville fut finalement publié le 15 juin 2010, après une très longue attente. Il qualifiait "d'injustifiable » la décision des soldats de tirer à balles réelles, exonérait NICRA de toute responsabilité et affirmait qu'aucune des victimes n'était engagée dans une activité justifiant l'utilisation d'armes à feu contre elle par les soldats ${ }^{29}$. Le même jour, le premier ministre conservateur David Cameron fit un discours dans lequel il présentait ses excuses au nom du gouvernement britannique ${ }^{30}$. L'accueil globalement positif que reçurent ces excuses ne contredit cependant pas les déclarations antérieures des membres des familles: en effet, les excuses n'ont été qu'une sorte d'épilogue à l'enquête, et non une solution de remplacement comme il en avait été question sous le gouvernement Blair.

La recherche de vérités consensuelles sur lesquelles bâtir une nouvelle société viable et pacifiée est un exercice auquel se livrent souvent les sociétés dites « en transition». Elle est parfois confiée à des " organismes de transition ", dont l'exemple le plus célèbre est sans doute la "Commission pour la Vérité et la Réconciliation" établie par Nelson Mandela en Afrique du Sud à la fin de l'apartheid ${ }^{31}$. Ces organismes ont toujours pour but, comme l'explique Martin Imbleau, « d'établir une vérité, une vérité historique qui servira de référence pour le présent comme pour le futur», car "aucune société, aucune transformation ne peut se construire sur des affirmations empiriques contestées concernant la vérité historique ${ }^{32} »$. L'établissement de cette vérité doit tout d'abord jouer un rôle d'éducation, afin de ne pas répéter les erreurs passées. Elle doit aussi permettre de rétablir la confiance des citoyens en leur gouvernement : lorsque le gouvernement a été impliqué dans les violences, l'appareil d'État peut avoir besoin de montrer sa bonne volonté et son intégrité, afin de permettre à la société de se reconstruire sur des bases saines. Enfin, elle doit surtout apporter une « résolution ${ }^{33}$ » : il faut « refermer » le passé, refermer les blessures qu'il a pu laisser, ou encore clore les débats qu'il suscite, afin de pouvoir passer à autre chose. L'enquête Saville, bien elle n'ait pas eu, contrairement aux commissions pour la vérité, l'ambition de passer en revue tous les événements contentieux d'une longue période de conflit, avait bien pour but d'établir une vérité officielle sur un événement controversé du passé afin de faciliter la réconciliation dans le contexte d'un processus de paix, et peut donc être considérée à ce titre comme un organisme de transition.

Ce type d'enquête est toutefois loin de faire l'unanimité : ne devrait-on pas plutôt oublier le passé afin de préserver l'avenir ? Les partisans de cette solution ont souvent tendance à considérer les organismes de transition comme des exercices inutiles, voire néfastes, qui ne font que raviver les vieilles querelles sur le passé. Le journaliste Max Hastings, par exemple, raille l'enquête Saville qu'il qualifie « d'exercice d'archéologie ${ }^{34}$ ». 
dant, cette approche est critiquable dans le sens où elle semble rejeter les Troubles dans le passé, et même dans un passé lointain, alors que beaucoup des événements traumatiques susceptibles de nuire au processus de paix se sont produits dans un passé en réalité très récent. Lors de la commémoration de Bloody Sunday de 2009, le député du SDLP Alban Maginness a rappelé encore une fois que « ce qui était le passé pour certains était encore le présent pour d'autres ${ }^{35} »$. On peut raisonnablement penser que ces propos s'adressaient implicitement à ceux qui critiquent la BSJC et l'enquête Saville en arguant qu'il est ridicule de continuer à débattre d'un événement vieux de plus de trente ans.

En juin 2007, notamment, deux déclarations ont provoqué un tollé de la part des représentants des partisans de la nouvelle enquête. Tout d'abord, Maurice Hayes, homme politique et auteur de nombreux rapports et articles sur l'Irlande du Nord, a vivement critiqué les enquêtes publiques lors d'une conférence organisée sur le campus de Magee College le 4 juin 2007, affirmant qu'elles mettaient en danger la réconciliation en «rouvrant de vieilles plaies ", en "réveillant de vieux fantômes» et en "ravivant les vieilles animosités et les vieux soupçons ». Quelques jours plus tard, lors de l'Assemblée Générale des Presbytériens, le pasteur presbytérien John Dunlop attaquait lui aussi l'enquête Saville, affirmant que de telles enquêtes risquaient de « déstabiliser le futur » et devraient être closes «immédiatement ${ }^{36}$ ». Ces déclarations ont été très mal accueillies par les familles des victimes de Bloody Sunday: John Kelly les a qualifiées « d'insultantes », ajoutant que de tels propos sapaient le travail effectué par la BSJC depuis des années ${ }^{37}$.

demandes de la BSJC représentent en effet la tendance inverse, qui consiste à penser que pour se libérer du passé, il faut d'abord s'y confronter. Martin Finucane, membre de la Irish Campaign for Truth, explique: «Si nous devons surmonter notre passé, il faut d'abord que nous arrivions à l'accepter, et nous ne pouvons faire cela que si nous savons la vérité sur notre passé ${ }^{38}$. " Là où d'autres invoquent la nécessité de préserver la confiance mutuelle entre les communautés comme une raison de ne pas faire ressurgir les fantômes du passé, Fionnuala Ni Aolain, dans son livre sur la violence d'État en Irlande du Nord, considère qu'il y a dans les sociétés en transition « un besoin d'affirmer des vérités, [...] en partie pour rétablir un peu de civilité dans une société civile déchirée, permettant de cette manière d'aller vers une certaine réconciliation entre les communautés ${ }^{39} »$. Les organismes de transition ne sont donc pas nocifs, mais nécessaires au processus de réconciliation: afin de pouvoir construire un avenir commun, il est indispensable d'arriver à un certain consensus sur le passé. Il ne s'agit pas de considérer le passé comme un fardeau dont on doit se débarrasser, mais de travailler sur lui afin de l'empêcher de nuire au futur, ou comme le dit élégamment Bill Rolston, de réussir à « tourner la page sans refermer le livre ${ }^{40} \%$.

29 Souvent associée à la vérité, en particulier dans le discours de la BSJC, la notion de justice pose également problème : qu'entend-on exactement par «justice »? Selon le philosophe W. James Booth, le procès constitue " le côté rétribution de la mémoire-justice ${ }^{41}$ ", mais le rétablissement de la vérité est aussi en soi une forme de justice: «la justice rétablit la vérité morale du passé42. . On pourrait donc distinguer deux types de justice : la justicevérité, qui consisterait à laver le nom des victimes des soupçons formulés par le rapport Widgery, et la justice-rétribution, qui consisterait à entamer des poursuites judiciaires pour meurtre contre les soldats après la publication du rapport. Les membres de la BSJC, bien que des dissensions internes existent à ce sujet, ont choisi de demander dans leurs revendications officielles à la fois la justice-vérité et la justice-rétribution. 
ans le cadre d'une enquête ou d'une commission pour la vérité, ces deux objectifs de vérité et de justice peuvent parfois se nuire mutuellement. Comme le signale Bill Rolston :

[...] la vérité et la justice ne vont pas forcément de pair. Comme les événements dans les pays qui ont eu des commissions pour la vérité l'on montré, la vérité ne requiert pas forcément que l'objectif de justice soit complètement atteint, surtout en ce qui concerne les poursuites ${ }^{43}$.

31 La plupart des organismes de transition chargés de dire la vérité sur un ou plusieurs événements sont confrontés à ce dilemme. La peur des poursuites judiciaires constitue souvent un obstacle de taille lorsqu'il s'agit de faire la lumière sur les agissements de tous les protagonistes; doit-on pour autant décider de garantir aux personnes acceptant de parler une immunité contre les poursuites? La recherche de la vérité et la recherche de la justice-rétribution deviennent alors incompatibles, puisque l'une nécessite le sacrifice de l'autre. Bill Rolston analyse dans Turning the Page Without Closing the Book: The Right To Truth in the Irish Context plusieurs cas de commissions pour la vérité, notamment celui de l'Argentine. La junte militaire y avait passé une loi d'amnistie avant de quitter le pouvoir. Devant « la vague populaire de répudiation des militaires et le soutien pour la vérité et la justice $^{44} »$, le nouveau président Alfonsin décida de passer outre cette loi et fit emprisonner plusieurs officiers de haut rang. Cependant, il dut vite céder face aux menaces de coup d'État venant de l'armée, et toutes les personnes emprisonnées furent finalement amnistiées. Rolston écrit à propos de l'Argentine : « certains affirment que cet échec était inévitable, à partir du moment où la recherche de la vérité et la recherche de poursuites judiciaires étaient couplées ${ }^{45}$ \%. La Truth and Reconciliation Commission en Afrique du Sud a, pour sa part, préféré sacrifier la possibilité de poursuites judiciaires :

$\mathrm{Au}$ vu de la nature des accords négociés il était impossible d'entreprendre des poursuites judiciaires à grande échelle. Dans ce contexte, l'amnistie ne peut qu'être vue comme une pré condition nécessaire et inévitable aux accords de paix ${ }^{46}$.

L'enquête Saville a adopté une position que l'on pourrait qualifier d'intermédiaire : si aucun témoin ne s'est vu octroyer une immunité contre les poursuites, le tribunal a cependant décidé que les informations données par les témoins dans le cadre de l'enquête ne pourraient pas être utilisées contre eux par la suite lors d'éventuelles poursuites judiciaires ${ }^{47}$. Le communiqué de presse du 25 février 1999 explique les raisons de cette décision:

Lord Saville a demandé cette garantie car les témoins [...] auraient normalement le droit de refuser de répondre à des questions s'il y avait un risque d'autoincrimination. [...] Une telle assurance était nécessaire pour assister le tribunal dans sa tâche de recherche de la vérité concernant les événements de Bloody Sunday ${ }^{48}$.

Le Procureur général a déclaré avoir pris cette décision « dans l'intérêt général », après avoir estimé que "l'efficacité de l'enquête [...] serait probablement augmentée plutôt que diminuée» par cet arrangement ${ }^{49}$. On peut donc penser qu'il s'agit d'un choix de privilégier la vérité par rapport à la justice, ce qui est cohérent au vu des termes dans lesquels l'enquête a défini sa propre mission, mais ne sera pas suffisant pour satisfaire toutes les demandes de la BSJC puisque les témoignages recueillis ne pourront être utilisés par eux pour atteindre leur objectif de justice-rétribution.

On peut donc se demander dans quelle mesure un exercice comme l'enquête Saville est susceptible de remplir sa fonction d'apaisement: si elle a effectivement servi de tribune pour les personnes n'ayant pas pu s'exprimer pendant l'enquête Widgery, avec un nombre considérable de témoins entendus et des transcriptions consultables par tous sur Internet, et si la publication du rapport a fourni aux télévisions des images de liesse 
populaire et de familles soulagées d'obtenir enfin une déclaration d'innocence pour leurs proches, il est encore beaucoup trop tôt pour parler de succès.

Passée l'euphorie du 15 juin 2010, il apparaît que tous les activistes de la campagne pour la justice n'ont pas été convaincus par la publication du rapport Saville. Jim Collins, artiste basé à Derry et ayant participé à l'organisation des commémorations de Bloody Sunday depuis 1989, considère qu'on «ne peut pas parler de victoire». Selon lui, la publication du rapport a été avant tout un exercice de communication habilement « chorégraphié » par le gouvernement britannique : les médias étaient venus en masse le jour de la publication pour immortaliser le moment où les familles sortiraient du Guildhall, et les expressions de joie dues à la fin de l'attente et à la satisfaction d'avoir obtenu une déclaration d'innocence pour leurs proches. Jim Collins évoque le caractère trompeur de l'image de la foule devant le Guildhall applaudissant le discours de David Cameron: selon lui, elle montre les habitants de Derry, une ville majoritairement nationaliste, comme de "bons sujets britanniques applaudissant leur Premier Ministre ", alors qu'ils applaudissaient en réalité les excuses du gouvernement, et non l'institution. Il ajoute que, les familles n'ayant eu que quelques heures le 15 juin pour parcourir les conclusions principales d'un rapport d'environ 5000 pages, il est fort possible qu'elles découvrent "après réflexion » que certains aspects du rapport ne sont pas satisfaisants, mais il sera alors trop tard car l'affaire sera considérée comme classée par les médias ${ }^{50}$.

En effet, très vite après la publication du rapport, la famille de Gerry Donaghey a exprimé son mécontentement à propos de l'interprétation donnée par Saville d'un des incidents les plus controversés de Bloody Sunday: le problème des bombes à clous retrouvées sur le corps de Donaghey par la police. Selon la famille de ce dernier, et la majorité des témoins, Donaghey n'avait pas de bombes sur lui, et elles auraient été délibérément placées sur son corps après sa mort par des soldats, dans le but de l'incriminer. Le rapport Saville refuse de trancher sur ce cas, mais affirme malgré tout que Donaghey était « probablement en possession des bombes au moment où il a reçu la balle ${ }^{51}$ ». Bien qu'il prenne soin de préciser que Donaghey s'enfuyait lorsqu'on lui a tiré dessus, et que l'action du soldat était donc injustifiée, ce refus de l'exonérer totalement sur la question des bombes à clous a quelque peu terni la déclaration d'innocence le concernant: pour sa sœur Mary Donaghey, « [Saville] a donné avec une main et repris avec l'autre ${ }^{52}$ ».

Enfin, au rang des griefs qui subsistent du côté de certains membres des familles, se trouve la très faible probabilité de pouvoir engager des poursuites judiciaires contre les soldats. Dans une émission de la chaîne irlandaise TV3, le 24 septembre 2010, Gerry Duddy, membre de la BSJC, a réaffirmé son désir d'obtenir des poursuites judiciaires contre les soldats ${ }^{53}$. Cependant, cet objectif semble fort compromis, d'une part en raison de l'arrangement évoqué précédemment entre l'enquête et les témoins, et d'autre part parce qu'après le grand événement de la publication du rapport, il existe un sentiment souvent exprimé par la presse que des poursuites ne feraient que compromettre l'apaisement apporté par Saville, repousser encore la résolution du problème, et raviver les tensions intercommunautaires ${ }^{54}$.

Dans un contexte politique toujours très polarisé, la réhabilitation des victimes de Bloody Sunday reste souvent perçue comme une cause catholique ou nationaliste, et la réception du rapport Saville du côté protestant a été mitigée. On a pu assister à des gestes de sympathie intercommunautaire, dont le plus symbolique est probablement la venue de plusieurs dignitaires des Églises protestantes dans le Bogside pour rencontrer les familles des victimes de Bloody Sunday, le lendemain de la publication du rapport Saville. Cette 
décision a été saluée par les journaux de tous bords comme un geste porteur d'espoir et un symbole de réconciliation ${ }^{55}$.

Toutefois, la publication du rapport a également provoqué des réactions moins positives : Graham Dawson expliquait en 2007 que pour beaucoup d'unionistes, l'enquête Saville n'était qu'un "pot-de-vin » accordé à la communauté nationaliste pour acheter leur soutien aux accords de paix ${ }^{56}$. Dès 2004, on pouvait lire sur une fresque unioniste du quartier de Shankill à Belfast, photographiée par Jonathan McCormick : "Où sont nos enquêtes? Où est notre vérité ? Où est notre justice ${ }^{57}$ ?", allusion à peine voilée à l'enquête Saville. La publication très médiatisée du rapport a donc relancé le débat sur l'existence d'une « hiérarchie des victimes »: dans le contexte d'un conflit qui a fait plus de trois mille morts, certains considèrent que dépenser près de 200 millions de livres pour une enquête sur la mort de "seulement" treize personnes constitue une injustice profonde vis-à-vis des autres victimes. Alors que beaucoup de proches de victimes du terrorisme ont dû se faire une raison et renoncer à obtenir la justice, voire même la vérité, soit parce qu'une enquête était impossible, soit en raison de la libération des prisonniers politiques dans le cadre de l'Accord du Vendredi Saint, les victimes de l'Etat apparaissent parfois comme une catégorie privilégiée. Après le 15 juin 2010, de nombreux articles ont été publiés qui contenaient des déclarations provenant de la communauté unioniste, principalement d'hommes politiques ou de représentants d'associations de victimes, dénonçant cette situation comme une injustice ou appelant à faire des enquêtes sur les tragédies de «leur» communauté afin de maintenir une certaine égalité de traitement ${ }^{58}$. Willie Fraser, de l'association de victimes FAIR (Families Acting for Innocent Relatives), a notamment déploré l'existence d'un «déséquilibre » dans le traitement des victimes, ajoutant que l'argent dépensé pour l'enquête Saville montrait que «certains morts des Troubles [étaient] plus égaux que d'autres [sic $]^{59}$ ».

Si la publication du rapport Saville a été perçue comme un moment historique, et généralement positif en raison des images de liesse populaire et des discours triomphants des familles des victimes, il est difficile de dire si elle a apporté une réelle résolution au trauma. Saville, en insistant pour examiner chaque détail malgré les critiques sur la longueur et le coût de l'enquête, a réussi à convaincre les familles de son sérieux, et la "vérité » qu'il a livrée dans son rapport a été globalement bien reçue, malgré quelques désaccords notamment sur le cas de Gerry Donaghey. Cependant, une fois le problème de la vérité écarté, reste le problème plus épineux de la justice : la question des poursuites judiciaires, qui a toujours divisé même à l'intérieur de la BSJC, se retrouve désormais au premier plan. Les questions restent finalement entières : l'enquête Saville a-t-elle apporté une "résolution ", ou a-t-elle ouvert la porte à la rétribution? Constitue-t-elle la fin du chapitre de Bloody Sunday, ou le début d'une série de campagnes pour l'ouverture de nouvelles enquêtes? Contribuera-t-elle à l'apaisement, ou simplement à nourrir les rancœurs sur le thème de la " hiérarchie des victimes »? Les membres de la BSJC, qui ont reçu de l'organisation non-gouvernementale irlandaise Rehab le titre de «People of the Year» le 11 septembre 2010, affirment qu'en ce qui les concerne "le temps des campagnes est révolu ${ }^{60} "$, mais des groupes réclamant des enquêtes sur d'autres événements tragiques des Troubles ont déjà saisi l'occasion de donner plus de visibilité à leur cause, parfois avec l'aide de membres de la BSJC : c'est le cas, par exemple, des familles des onze victimes du «massacre de Ballymurphy » de $1971^{61}$. Cependant, les mots de David Cameron dans son discours au parlement, lorsqu'il a affirmé qu'il n'y aurait 
"plus d'enquête coûteuses et à durée indéterminée portant sur le passée ${ }^{2}$ ", laissent entendre que l'enquête Saville risque fort de rester une exception.

\section{NOTES}

1. Eamonn McCann (dir.), The Bloody Sunday Inquiry: The Families Speak Out, Dublin, Pluto Press, 2006, p. 6. Toutes les traductions sont de l'auteur.

2. Graham Dawson, «Trauma, Place and the Politics of Memory : Bloody Sunday, Derry, 1972-2004 », History Workshop Journal, n 59, 2005, p. 161.

3. Dermot Walsh, Bloody Sunday and the Rule of Law in Northern Ireland, Basingstoke, Macmillan, 2000, p. 12.

4. Graham Dawson, Making Peace With the Past: Memories, Trauma and the Irish Troubles, Manchester, Manchester University Press, 2007, p. 148.

5. Dawson, 2007, p. 148-149.

6. Trisha Ziff (dir.), Hidden Truths : Bloody Sunday 1972, Santa Monica, Smart Art, 1998, p. 41.

7. The Rt. Hon. Lord Widgery, Report of the Tribunal appointed to inquire into the events on Sunday, 30 $0^{\text {th }}$ January 1972, which led to the loss of life in connection with the procession in Londonderry on that day, London, Her Majesty's Stationary Service, 1972, p. 98.

8. Widgery, p. 97.

9. Dawson, 2007, p. 120.

10. Ziff, p. 23, p. 125.

11. Joelle Gartner, "Anger at the Heel : The Legacy of Bloody Sunday », in Ziff, p. 125.

12. Seamus McKinney, « Bloody Sunday family dismisses Jackson admission », Irish News, 30 mai 2007, [www.nuzhound.com/articles/irish_news/arts2007] may30_family_dismisses_Jackson_admission.php].

13. Don Mullan, « Blood, White and Blue », in Ziff, p. 42-43.

14. Hansard 1803-2005. UK Parliament, volume 217, c. 485, 21 janvier 1993 ; volume 288, c. 781 W, 24 janvier 1997 ; volume 289, c. 495, 30 janvier 1997 ; volume 289, c. 505-506, 30 janvier 1997 [http://hansard.millbanksystems.com].

15. Hansard, volume 289, c. 506, 30 janvier 1997.

16. David A. Croker, "Truth Commissions, Transitional Justice, and Civil Society» in Robert I. Rotberg, Dennis Thompson, Truth v. Justice: The Morality of Truth Commissions, Princeton, Princeton University Press, 2000, p. 102.

17. Bill Rolston, Turning the Page Without Closing the Book, Dublin, Irish Reporter Publications, 1996, p. 13.

18. Widgery, p. 45.

19. Ibid., p. 58, p. 99.

20. Peter Pringle, « Exorcising Widgery », in Ziff, p. 58. 
21. Ziff, p. 23.

22. Maureen E. Mulvihill, "The Camera Does Not Lie: Revisiting Bloody Sunday ", New Hibernia Review, volume 6, n 4, 2002, p. 154.

23. Widgery, p. 7-8.

24. Kathy Marks, «Blair Issues Apology For Irish Potato Famine », The Independent, 2 juillet 1997, [www.independent.co.uk/news/blair-issues-apology-for-irish-potatofamine-1253790.html].

25. Jonathan Powell, Great Hatred, Little Room: Making Peace in Northern Ireland, Londres, Bodley Head, 2008, p. 45.

26. Joanne O'Brien, A Matter of Minutes: The Enduring Legacy of Bloody Sunday, Dublin, Wolfhound Press, 2002, p. 88.

27. Gartner in Ziff, p. 127.

28. Michael Cunningham, «Saying Sorry: The Politics of Apology », The Political Quarterly, volume 70, n 3, 1999, p. 287.

29. The Rt. Hon. Lord Saville of Newdigate, the Hon. William Hoyt, the Hon. John Toohey, "Report of the Bloody Sunday Inquiry", I.4.1, I.4.33, I.3.79, Bloody Sunday Inquiry Website, 17 juin 2010, [http://report.bloody-sunday-inquiry.org].

30. Discours de David Cameron, "House of Commons Hansard Debates ", vol. 511, c.730-742, 15 juin 2010, United Kingdom Parliament, [www.publications.parliament.uk/pa/ cm201011/cmhansrd/cm100615/debtext/100615-0004.htm].

31. Rolston, 1996, p. 12-14.

32. Martin Imbleau, «Initial Truth Establishment By Transitional Bodies and the Fight Against Denial », in William A. Schabas, Shane Darcy (dir.), Truth Commissions and Courts : The Tension Between Criminal Justice and the Search for Truth, Dordrecht, Kluwer Academic Publishers, 2004, p. 162.

33. «Closure » en anglais.

34. Max Hastings, "Give us inquiries into the banking crisis and Iraq, not Bloody Sunday ", The Guardian, 10 novembre 2008, [www.guardian.co.uk/commentisfree/2008/ nov/10/northern-ireland-banks].

35. Alban Maguinness, discours à l'occasion de la marche commémorative annuelle de Bloody Sunday, Derry, $1^{\mathrm{er}}$ février 2009.

36. Susan McKay, «The need for inquiries is all part of the peace process ", Irish News, 12 juin 2007, [http://www.nuzhound.com/articles/irish_news/arts2007/ jun12_need_for_inquiries__SMcKay.php].

37. Sarah Brett, « Outrage At Bloody Sunday 'Insult' », The Belfast Telegraph, 5 juin 2007, [ www.belfasttelegraph.co.uk/news/local-national/outrage-at-bloody-sunday-insult-13447528.html].

38. Brandon Hamber (dir.), Past Imperfect: Dealing With the Past in Northern Ireland and Societies in Transition, Derry/Londonderry, INCORE, 1998, p. 20.

39. Ibid., p. 11.

40. Bill Rolston, 2000.

41. William James Booth, Communities of Memory: On Witness, Identity and Justice, London, Cornell University Press, 2006, p. 135.

42. Ibid., p. 115. 
43. Bill Rolston, Unfinished Business: State Killings and the Quest For Truth, Belfast, Beyond The Pale, 2000, p. 325.

44. Rolston, 1996, p. 15.

45. Rolston, 1996, p. 16.

46. Hamber, p. 60.

47. "Attorney General Press Notice», Press Notices, Bloody Sunday Inquiry Web Site, 25 février 1999, [www.bloody-sunday-inquiry.org/press/Archive/25feb99.htm].

48. "Attorney General Press Notice», Press Notices, Bloody Sunday Inquiry Web Site, 25 février 1999, [www.bloody-sunday-inquiry.org/press/Archive/25feb99.htm].

49. Ibid.

50. Entretien avec Jim Collins, 22 juillet 2010, Derry/Londonderry.

51. The Rt. Hon. Lord Saville of Newdigate, the Hon. William Hoyt, the Hon. John Toohey, « Report of the Bloody Sunday Inquiry », VII.145.25, Bloody Sunday Inquiry Website, 17 juin 2010, [http://report.bloody-sunday-inquiry.org].

52. Alan Healy, « Victim's Family Angry At Nail Bombs Claim », Derry News, p. 5, 17 juin 2010.

53. Interview de Gerry Duddy, Ireland AM, TV3, 24 septembre 2010, [www.tv3.ie/ videos.php?

video=27359\&locID=1.65.74\&date=2010-09-24\&date_mode=\&page=1\&show_cal=2\&newspanel=\&showspanel=\&web_only=\&full_episodes= ].

54. "Justice and Truth Have Finally Been Served», The Independent, 16 juin 2010, [ www.independent.co.uk/opinion/leading-articles/justice-and-truth-have-finally-been-

served-2001515.html] ; « Bloody Sunday : Prosecutions Would Be In No One's Interest », The Telegraph, 15 juin 2010, [www.telegraph.co.uk/comment/telegraph-view/7831212/BloodySunday-Prosecutions-would-be-in-no-ones-interest.html]; Max Hastings, "This Grossly Misguided Excavation of the Past », The Daily Mail, 17 juin 2010, [www.dailymail.co.uk/news/ article-1286953/MAX-HASTINGS-This-grossly-misguided-excavation-past.html].

55. «Protestant Church Leaders Extend 'Hand of Friendship' To Bloody Sunday Families ", Derry Journal, 16 juin 2010, [www.derryjournal.com/journal/Protestant-churchleaders-extend-6364802.jp] ; Brian Hutton, "Protestant Leaders Visit the Bogside», The Newsletter, 17 juin 2010, p. 4 ; "Protestant Church Leaders To Visit Bloody Sunday Site ", The Belfast Telegraph, 16 juin 2010, [www.belfasttelegraph.co.uk/news/local-national/bloodysunday/protestant-church-leaders-to-visit-bloody-sunday-site-14843810.html\#ixzzOr9NxiMl2];

John Cooney and George Jackson, « Historic 'Hands Across Divide' As Church Leaders and Families Unite », The Independent, 17 juin 2010, [www.independent.ie/national-news/historichands-across-divide-as-church-leaders-and-families-unite-2223358.html].

56. Dawson, 2007, p. 202.

57. Jonathan McCormick, «30 Years of Indiscriminate Slaughter by So-Called NonSectarian Irish Freedom Fighters" in "A Directory of Murals ", Cain Web Service, album 59, photo 2062, mars 2004, [http://cain.ulst.ac.uk/mccormick/album59.htm].

58. Sam Lister, "Tearful McCrea Names 'Forgotten Victims' of Provos", The Belfast Telegraph, 7 juillet 2010, [www.belfasttelegraph.co.uk/news/politics/tearful-mccrea-nameslsquoforgotten-victimsrsquo-of-provos-14867409.html\#\#ixzz0tHjb4gjh]; “Campbell Demands Answers On Deaths", The Londonderry Sentinel, 24 juin 2010, [www.londonderrysentinel.co.uk/ news/Campbell-demands-answers-on-deaths.6381635.jp]; Sir Reg Empey, "Inquiries Are No 
Way To Build a Shared Future", The Guardian, 15 juin 2010, [www.guardian.co.uk/uk/2010/ jun/15/ulster-unionist-sir-reg-empey].

59. Henry McDonald, «Bloody Sunday : $£ 191 \mathrm{~m}$ and 434 Days - Will Saville Draw a Line Under Past? », The Guardian, 11 juin 2010, [www.guardian.co.uk/uk/2010/jun/11/bloodysunday-saville-draw-line].

60. Interview de Gerry Duddy, Ireland AM, TV3, 24 septembre 2010, [www.tv3.ie/videos.php? video=27359\&locID=1.65.74\&date=2010-09-24\&date_mode=\&page=1\&show_cal=2\&newspanel=\&showspanel=\&web_only=\&full_episodes= ].

61. «Ballymurphy massacre probe call backed», The Belfast Telegraph, 21 septembre 2010, [www.belfasttelegraph.co.uk/news/local-national/northern-ireland/ballymurphy-massacreprobe-call-backed-14954315.html\#ixzz10LkVwIgK].

62. Discours de David Cameron, «House of Commons Hansard Debates », vol. 511, c.741, 15 juin 2010, United Kingdom Parliament, [www.publications.parliament.uk/pa/cm201011/ cmhansrd/cm100615/debtext/100615-0004.htm].

\section{RÉSUMÉS}

Bloody Sunday reste l'un des événements des Troubles en Irlande du Nord qui a le plus marqué la mémoire collective. Son potentiel traumatique s'explique d'une part par l'implication des forces de l'État, et d'autre part, par l'impact doublement traumatisant du rapport Widgery : la première enquête publique de 1972 fut perçue comme une tentative de blanchir l'armée. Après des années de campagne, les familles obtinrent en 1998 une seconde enquête. Dans un contexte de réconciliation, un des objectifs de l'enquête Saville était de "guérir " les blessures laissées par Bloody Sunday et par le rapport Widgery. Cependant, comme tous les organismes de transition dont le rôle est d'apaiser les tensions liées à un passé contentieux afin de permettre aux communautés concernées de construire un futur commun, l'enquête a également suscité doutes et critiques.

Bloody Sunday is among the events of the Northern Irish Troubles which have left a deep imprint on popular memory. Its traumatic potential can be explained on the one hand by the involvement of the state forces, and on the other hand, by the additional traumatic impact of the Widgery report: the 1972 public inquiry was perceived as a "whitewash". In 1998, after years of campaigning by the families, the Saville Inquiry was set up. In a context of reconciliation, one of its objectives was to "heal" the wounds left by Bloody Sunday and by the Widgery Inquiry. However, like all transitional bodies, the role of which is to ease the tensions linked to a contentious past in order to allow the communities in question to build a common future, the Saville Inquiry has also raised criticism and doubt. 
INDEX

Keywords : Northern Ireland - Bloody Sunday, justice, reconciliation, Northern Ireland - conflict, Northern Ireland - post-conflict, Northern Ireland - peace process, trauma

Mots-clés : Irlande du Nord - Bloody Sunday, Irlande du Nord - conflit, Irlande du Nord processus de paix, Irlande du Nord - post-conflit

\section{AUTEUR}

\section{CHARLOTTE BARCAT}

Université de Brest-UBO 